\title{
An Appropriate Energy Intake Proportion of Three Major Nutrients for Treatment of Type 2 Diabetes
}

\author{
Hidekatsu Yanai ${ }^{\text {a, b}}$, Hisayuki Katsuyama ${ }^{a}$
}

\begin{abstract}
To keep good glycemic control and suppress the development of diabetic complication, diet therapy is a very crucial treatment for type 2 diabetes. Recently, the effectiveness of carbohydrate-restricted diet, and the diet for sarcopenia and frailty have been suggested, inducing the paradigm shift of diet for type 2 diabetes. There is insufficient evidence regarding the distribution of nutrients in the diet of patients with type 2 diabetes. Here, we reviewed the current state of evidences and guidelines regarding energy allocation of nutrients for patients who have already developed diabetes. At present, any Japanese and international guidelines did not clearly describe an appropriate energy intake proportion of three major nutrients for treatment of type 2 diabetes. The tailor-made medicine should also be applied to the diet for type 2 diabetes. When setting the nutritional balance of diabetic patients, first, we should fully understand each patient's current situation and make appropriate adjustments, taking into the account of changes in body weight, blood glucose and lipid levels, patient preferences, and feasibility.
\end{abstract}

Keywords: Type 2 diabetes; Diet therapy; Nutrients; Energy intake; Carbohydrate

\section{Introduction}

The incretin-related drugs and sodium-glucose cotransporter-2 (SGLT2) inhibitors appeared in the treatment of type 2 diabetes, and pharmacological therapy for type 2 diabetes has made remarkable progress. To keep good glycemic control and suppress the development of diabetic complication, diet and exercise are very crucial treatment for type 2 diabetes. Recently, the effectiveness of carbohydrate-restricted

Manuscript submitted September 14, 2020, accepted October 5, 2020

Published online October 16, 2020

aDepartment of Diabetes, Endocrinology and Metabolism, Kohnodai Hospital, National Center for Global Health and Medicine, Chiba, Japan

${ }^{\mathrm{b}}$ Corresponding Author: Hidekatsu Yanai, Department of Diabetes, Endocrinology and Metabolism, Kohnodai Hospital, National Center for Global Health and Medicine, 1-7-1 Kohnodai, Ichikawa, Chiba 272-8516, Japan. Email: dyanai@hospk.ncgm.go.jp

doi: https://doi.org/10.14740/jem693 diet, and the diet for sarcopenia and frailty have been suggested, inducing the paradigm shift of diet for diabetes.

First, we will summarize the current state of evidences and guidelines regarding energy allocation of nutrients. Second, we like to consider how to determine the energy allocation in diet therapy for diabetes. The diet to reduce the risk of diabetes in people without diabetes and the diet for patients who have already developed diabetes are sometimes confused. Here, we will describe the latter.

\section{Changes in Japanese Physique and Diet}

According to the 2016 National Health and Nutrition Survey by the Ministry of Health, Labor and Welfare, the number of people with diabetes reached 10 million, and the increase has not been stopped [1]. It has been considered that the increase in diabetic patients is influenced by the increase in obesity in addition to the progress of aging. According to the 2018 National Health and Nutrition Survey, the proportion of obese persons with a body mass index (BMI) of 25 $\mathrm{kg} / \mathrm{m}^{2}$ or more was $32.2 \%$ for men and $21.9 \%$ for women [2]. Although there has been no significant change over the last 10 years, the proportion of obese men has significantly increased as compared with those in the 1980s. The increase in obese people is linked to the increase in diabetic patients via insulin resistance. Compared with Westerners, Asians have insulin secretion insufficiency, and even mild obesity causes insulin resistance, leading to metabolic disorders such as hyperglycemia. Therefore, correcting obesity by diet is very important to improve glucose, lipids, and blood pressure and also to prevent diabetic complications. How is the Japanese diet changing? According to the National and Health Nutrition Survey, Japanese energy intake decreased from 2,184 kcal in 1965 to $1,897 \mathrm{kcal}$ in 2017 [2]. Among the three major nutrients, the proportion of carbohydratederived energy among total energy decreased from $72.1 \%$ to $57.5 \%$, while the fat-derived energy increased from $14.8 \%$ to $27.7 \%$. There is no significant change in protein energy proportion from $13.1 \%$ to $14.6 \%$, however, the intake of vegetable protein has been decreasing and the intake of animal protein has been increasing [2]. It is speculated that such changes in Japanese eating habits as well as physical activity declines have a large effect on the increase in obese people. However, it remains unclear what dietary changes increase obesity. 


\section{The Diet Therapy for Type 2 Diabetes in Japan}

In Japan, first, we determine total dietary energy intake and then allocating total energy into the three major nutrients. The total energy intake is determined by multiplying the standard weight by a coefficient based on the amount of physical activity (light exertion: $25-30 \mathrm{kcal}$, normal exertion: $30-35$ kcal, heavy exertion: $>35 \mathrm{kcal}$ ), and the carbohydrate-derived energy is in the range of $50 \%$ or more and $60 \%$ or less, proteinderived energy is 1.0 to $1.2 \mathrm{~g} / \mathrm{kg}$ body weight/day, and the rest is fat-derived energy. Saturated fatty acids (SFA)- and polyunsaturated fatty acids (PUFA)-derived energy should be within $7 \%$ and $10 \%$ of total intake energy. Recently, it has been reconfirmed that there is insufficient evidence regarding the energy allocation of nutrients, and the need for flexible consideration for each patient is being emphasized.

\section{Evidences on Carbohydrate Intake for Type 2 Diabetes Management}

In the randomized controlled trial (RCT) where 24 Japanese type 2 diabetic patients divided into two groups, an energy-restricted diet group and a $70-130 \mathrm{~g} /$ day carbohydrate-restricted diet group for 6 months, hemoglobin A1c (HbA1c) and triglyceride was lower in the carbohydrate-restricted diet group [3]. In the RCT using 33 Japanese type 2 diabetic patients, a mild carbohydrate-restricted diet of $130 \mathrm{~g}$ /day was performed for 6 months, BMI and $\mathrm{HbAlc}$ were significantly improved in a mild carbohydrate-restricted diet group [4]. However, in this study, energy intake was also reduced in the carbohydrate-restricted group, and the difference in BMI and $\mathrm{HbAlc}$ between the two groups disappeared in the follow-up 1 year later [5]. Carbohydrate restriction for type 2 diabetic patients may have a certain effect in the short term, but its long-term efficacy and safety are not fully elucidated.

The Dietary Intervention Randomized Control Trial (DIRECT) study was conducted for 2 years by dividing 332 obese patients including patients with type 2 diabetes, into low-carbohydrate diet, low-fat diet, and Mediterranean diet [6]. Lowcarbohydrate diet and Mediterranean diet group showed significantly greater reduction in body weight and improvement of insulin resistance and lipid profile. However, total energy intake was also reduced in low-carbohydrate diet group, and weight loss was also observed in low-fat diet group. A followup study showed significant weight rebound in low-carbohydrate diet group [7]. From these results, the effect seen in low-carbohydrate diet group may be related to the decrease in energy intake, and it cannot be concluded that the effect is due to carbohydrate restriction, and it may be difficult to carry out carbohydrate restriction in the long term.

The meta-analysis of $10 \mathrm{RCTs}$ showed that $\mathrm{HbAlc}$ was significantly lower for the first year in the low-carbohydrate diet group with carbohydrate content of less than $45 \%$ of total energy [8]. After 1 year, the difference in HbAlc disappeared, and no difference in BMI or low-density lipoprotein cholesterol (LDL-C) was observed. In a cohort study of 15,428 general population, there was a J-shaped correlation between carbohydrate-derived energy intake proportion and the overall mortality rate, and the lowest overall mortality was observed in population whose carbohydrate-derived energy intake proportion were $50-55 \%$ [9]. In a cohort study of 1,516 Japanese type 2 diabetic patients, no significant correlation was found between baseline carbohydrate intake and the risk of developing nephropathy, retinopathy, or cardiovascular disease [10].

The long-term effects of carbohydrate intake in people with type 2 diabetes remain unclear. Furthermore, it is necessary to pay attention to the quality of carbohydrates. Japanese and international guidelines recommend reducing intake of refined carbohydrates and added sugars and increasing intake of dietary fiber.

\section{Evidences on Protein Intake for Type 2 Diabe- tes Management}

It has been reported that increased intake of protein, especially animal protein, is a risk of developing diabetes and increases insulin resistance. However, the association of protein intake with glycemic control and development of complications in patients with type 2 diabetes remains unknown. A cohort study of 8,461 general populations without renal disease followed for 6.4 years showed compared with intermediate protein intake, individuals with either higher or lower protein intake had higher cardiovascular event rates [11]. All-cause mortality and non-cardiovascular mortality also were significantly associated with protein intake; individuals with low protein intake had the highest event rates. No association between baseline protein intake and rate of renal function decline during followup was observed [11]. A meta-analysis of eight RCTs using patients with diabetic nephropathy showed that protein intake of $0.6-0.8 \mathrm{~g} / \mathrm{kg} /$ day reduced proteinuria, but no change in estimated glomerular filtration rate (eGFR) [12]. However, another meta-analysis of $13 \mathrm{RCTs}$, protein intake of 0.6 to $0.8 \mathrm{~g} /$ $\mathrm{kg} /$ day was associated with improved eGFR in patients with diabetic nephropathy [13].

There is insufficient evidence regarding the optimal protein intake in type 2 diabetes, and there is controversy regarding the effect of protein restriction on the prevention of diabetic nephropathy. Recently, the importance of protein intake has been emphasized in elderly diabetic patients from the viewpoint of preventing sarcopenia and flail. In a 3-year follow-up study of elderly female diabetic patients, physical function was maintained in the group receiving protein intake of $1.0 \mathrm{~g} / \mathrm{kg} /$ day or more compared to the group receiving less than $1.0 \mathrm{~g} /$ $\mathrm{kg} /$ day [14]. It was also reported that Japanese chronic kidney disease (CKD) patients over the age of 65 had a lower risk of death in the highest protein intake group [15].

\section{Evidences on Fat Intake for Type 2 Diabetes Management}

There is no definitive view on the effect of total intake of fat in diabetic patients. Recently, the type of fat has become more important than the total intake of fat. The DIRECT study 
Table 1. Descriptions on Energy Intake Proportion of Three Major Nutrients for Treatment of Type 2 Diabetes in Japanese and International Guidelines

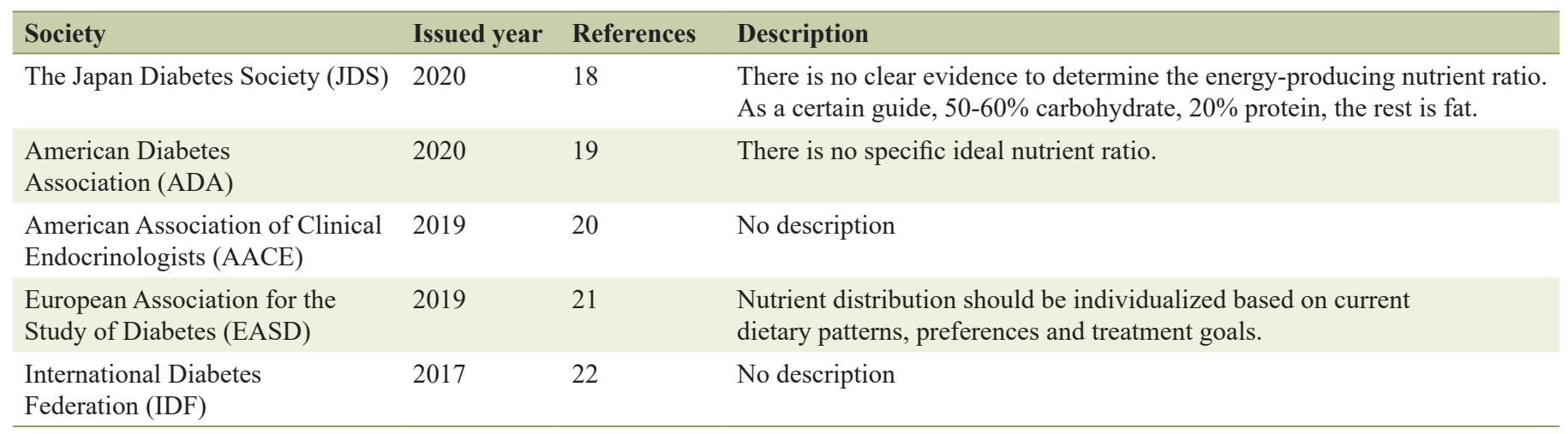

has shown that the Mediterranean diet group, which contains a large amount of PUFA and mono-unsaturated fatty acids (MUFA), showed more beneficial effect on weight loss and serum lipid profile as compared with the low-fat diet group [6]. In the meta-analysis of nine RCTs using patients with abnormal glucose metabolism, although there was no change in insulin resistance, $\mathrm{HbAlc}$ was lower in the group with high intake of PUFA [16].

There are disagreements about the relationship between the intake of fish-derived n-3 fatty acids and glycemic control. RCTs using eicosapentaenoic acid (EPA) or docosahexaenoic acid (DHA) have not shown prevention of cardiovascular events [17]. At present, the effects of n-3 fatty acids on type 2 diabetic patients have not been confirmed.

\section{Evidences and Guidelines for Energy Intake Proportion of Three Major Nutrients for Type 2 Diabetes}

There is little evidence on the proper balance of the three major nutrients. The nutrient balance varies greatly depending on the region. Compared to Europe and the USA, Japanese intake larger energy from carbohydrates, but smaller energy from fats. Furthermore, it is very difficult to clarify the proper nutrient balance because the intakes of the three major nutrients are closely related to each other.

Table 1 shows the current status of the description of nutrient balances in Japanese and international guidelines [18-22]. According to the guidelines of the American Diabetes Society (ADA), there are no specific ratios of the three major nutrients in diabetic patients, and it is necessary to individually set specific dietary contents while considering total energy intake and treatment goals, and specific nutrient balance is not described [19]. In the Dietary Reference Intake for Japanese (2020 edition), the standards for nutrient balance in adults are $50-65 \%$ carbohydrates, $13-20 \%$ proteins, and $20-30 \%$ fats (SFA $7 \%$ or less) [23]. In the current situation where there is insufficient evidence regarding the proper nutrient balance in diabetic patients, it is necessary to consider the intake status of healthy people as a reference. In the "Proposal for Diet of the Japan
Diabetic Society" issued in 2013, carbohydrates 50-60\%, protein $20 \%$, and the rest are fats in the total energy intake, and if the fats exceed $25 \%$, PUFA should be increased [24]. In the guideline of the Japan Diabetes Society in 2020 [18], it was a step back expression that "There is no clear evidence to set the desirable energy-producing nutrient ratio for the prevention and management of diabetes". It is said that the energy ratio indicated in the proposal is "a certain guide". Furthermore, a statement that "We should respond flexibly according to the amount of physical activity of the patient, the state of complications, age, preference, etc." was added, emphasizing the need to set individually for each patient.

\section{How to Set Nutrient Distribution for Patients With Type 2 Diabetes}

Eating habits are influenced not only by the area in which they were born and raised, but also by various factors such as family environment, economic conditions, tastes, and age. It has been argued that Japanese food is an ideal diet for Japanese diabetic patients, but in reality, the Japanese diet is diversified and there are large individual differences. In addition, the Diabetes Treatment Guide added that the treatment goal for diabetes is "to allow diabetic patients to live the same life as healthy people without suffering from stigma and social disadvantages caused by diabetes". Given that, despite lack of sufficient evidence, the fact that patients are forced to have a uniform diet because of diabetes and that they are not allowed to enjoy a variety of dietary cultures may be far from the ideal treatment for diabetes.

In the 2020 edition of Dietary Reference Intake for Japanese, while protein has a required amount among the three major nutrients, it is unlikely that carbohydrates will fall below the required amount except under special conditions. It is appropriate to first determine the amount of protein, then the amount of fat, and the remainder as carbohydrate [23]. When setting the nutrient balance in dietary guidance for diabetic patients, it may be useful to refer to this way of thinking rather than simply allocating the amount of energy intake among the three major nutrients. In addition, the nutrient balance shown 
in the "Recommendations for Diet of the Japan Diabetes Society" can be helpful in the cases with extremely unbalanced diet [24]. The tailor-made medicine is not limited to drug therapy. When setting the nutritional balance of diabetic patients, first fully understand each patient's current situation and make appropriate adjustments, taking into the account of changes in body weight, blood glucose and lipid, patient preferences, and feasibility.

\section{Acknowledgments}

None to declare.

\section{Financial Disclosure}

Authors have no financial disclosure to report.

\section{Conflict of Interest}

The authors declare that they have no conflict of interest concerning this article.

\section{Informed Consent}

Not applicable.

\section{Author Contributions}

H.K. collected literature and H.Y. wrote the manuscript. Both authors approved the final paper.

\section{Data Availability}

The authors declare that data supporting the findings of this study are available within the article.

\section{References}

1. Ministry of Health, Labor and Welfare. 2016 "National Health and Nutrition Survey".

2. Ministry of Health, Labor and Welfare. 2018 "National Health and Nutrition Survey".

3. Yamada Y, Uchida J, Izumi H, Tsukamoto Y, Inoue G, Watanabe Y, Irie J, et al. A non-calorie-restricted lowcarbohydrate diet is effective as an alternative therapy for patients with type 2 diabetes. Intern Med. 2014;53(1):1319.

4. Sato J, Kanazawa A, Makita S, Hatae C, Komiya K, Shimizu T, Ikeda F, et al. A randomized controlled trial of $130 \mathrm{~g} /$ day low-carbohydrate diet in type 2 diabetes with poor glycemic control. Clin Nutr. 2017;36(4):992-1000.
5. Sato J, Kanazawa A, Hatae C, Makita S, Komiya K, Shimizu T, Ikeda F, et al. One year follow-up after a randomized controlled trial of a $130 \mathrm{~g} /$ day low-carbohydrate diet in patients with type 2 diabetes mellitus and poor glycemic control. PLoS One. 2017;12(12):e0188892.

6. Shai I, Schwarzfuchs D, Henkin Y, Shahar DR, Witkow S, Greenberg I, Golan R, et al. Weight loss with a low-carbohydrate, Mediterranean, or low-fat diet. N Engl J Med. 2008;359(3):229-241.

7. Schwarzfuchs D, Golan R, Shai I. Four-year followup after two-year dietary interventions. N Engl J Med. 2012;367(14):1373-1374.

8. Snorgaard O, Poulsen GM, Andersen HK, Astrup A. Systematic review and meta-analysis of dietary carbohydrate restriction in patients with type 2 diabetes. BMJ Open Diabetes Res Care. 2017;5(1):e000354.

9. Seidelmann SB, Claggett B, Cheng S, Henglin M, Shah A, Steffen LM, Folsom AR, et al. Dietary carbohydrate intake and mortality: a prospective cohort study and meta-analysis. Lancet Public Health. 2018;3(9):e419-e428.

10. Horikawa C, Yoshimura Y, Kamada C, Tanaka S, Tanaka $\mathrm{S}$, Matsunaga S, Hanyu $\mathrm{O}$, et al. Is the proportion of carbohydrate intake associated with the incidence of diabetes complications? An analysis of the Japan diabetes complications study. Nutrients. 2017;9(2).

11. Halbesma N, Bakker SJ, Jansen DF, Stolk RP, De Zeeuw D, De Jong PE, Gansevoort RT, et al. High protein intake associates with cardiovascular events but not with loss of renal function. J Am Soc Nephrol. 2009;20(8):17971804.

12. Pan Y, Guo LL, Jin HM. Low-protein diet for diabetic nephropathy: a meta-analysis of randomized controlled trials. Am J Clin Nutr. 2008;88(3):660-666.

13. Nezu U, Kamiyama H, Kondo Y, Sakuma M, Morimoto T, Ueda S. Effect of low-protein diet on kidney function in diabetic nephropathy: meta-analysis of randomised controlled trials. BMJ Open. 2013;3(5).

14. Rahi B, Morais JA, Gaudreau P, Payette H, Shatenstein B. Energy and protein intakes and their association with a decline in functional capacity among diabetic older adults from the NuAge cohort. Eur J Nutr. 2016;55(4):17291739.

15. Watanabe D, Machida S, Matsumoto N, Shibagaki Y, Sakurada T. Age modifies the association of dietary protein intake with all-cause mortality in patients with chronic kidney disease. Nutrients. 2018;10(11).

16. Schwingshackl L, Strasser B, Hoffmann G. Effects of monounsaturated fatty acids on glycaemic control in patients with abnormal glucose metabolism: a systematic review and meta-analysis. Ann Nutr Metab. 2011;58(4):290-296.

17. Risk, Prevention Study Collaborative Group, Roncaglioni MC, Tombesi M, Avanzini F, Barlera S, Caimi V, et al. n-3 fatty acids in patients with multiple cardiovascular risk factors. N Engl J Med. 2013;368(19):1800-1808.

18. The Japan Diabetes Society. Diabetes practice guidelines 2019. Nankodo, Japan.

19. American Diabetes A. 5. Facilitating behavior change and well-being to improve health outcomes: standards of medical care in diabetes-2020. Diabetes Care. 2020;43(Suppl 
1):S48-S65.

20. Garber AJ, Abrahamson MJ, Barzilay JI, Blonde L, Bloomgarden ZT, Bush MA, Dagogo-Jack S, et al. Consensus statement by the American Association of Clinical Endocrinologists and American College of Endocrinology on the comprehensive type 2 diabetes management algorithm - 2019 executive summary. Endocr Pract. 2019;25(1):69-100.

21. Cosentino F, Grant PJ, Aboyans V, Bailey CJ, Ceriello A, Delgado V, Federici M, et al. 2019 ESC Guidelines on diabetes, pre-diabetes, and cardiovascular diseases developed in collaboration with the EASD. Eur Heart J. 2020;41(2):255-323.

22. . IDF clinical practice recommendations for managing type 2 diabetes in primary care. 2017.

23. Ministry of Health, Labor and Welfare. Dietary reference intake for Japanese (2020 Edition).

24. The Japan Diabetes Society. Recommendations of the Japan diabetes society on the Japanese diabetic diet-current status and issues of the diet in diabetes. 2013. 